UDC 53.043

\title{
THE COMPLETE FORM OF THE PROPAGATION CONSTANT IN A NONINERTIAL REFERENCE FRAME FOR NUMERICAL ANALYSIS
}

\author{
K. M. Zeyde \\ Ural Federal University, Institute of radio electronics and information technologies, \\ Mira 19, Ekaterinburg 620002, Russia
}

The paper is received on March 25, 2019

\begin{abstract}
In this paper we present a derivation of the strict form of the propagation constant for a rotating disk, taking into account the inertial forces of the reference system. This work is a generalization of known facts in a complete methodology. We use formulations related to the use of deflection angle - transition of the ray from a straight-line trajectory under the action of inertia forces. This study allows conducting high-precision computer simulations of electromagnetic effects arising in non-inertial frames of reference. Strict conclusions are achieved by the exception of rest frame theory. Two-dimensional electromagnetic propagation problem is solved in a non-inertial frame of reference. A rotating frame of reference is a good example of any noninertial frame of reference, therefore, this particular model was chosen to be considered. We use the classical approach for describing the conditions for the propagation of electromagnetic waves in noninertial reference frames: the refined refractive index of the medium. The possibility of modeling using the obtained formulations is demonstrated on a rotating circular infinite cylinder. Verification of the results was carried out. Distributions of the propagation constant in a rotating medium are presented. The results are obtained using CAD of electromagnetic modeling. The comparison was made for data from open sources. Validation of the method proved its effectiveness.
\end{abstract}

Key words: propagation constant, noninertial reference frame, electromagnetic scattering, relativity.

\section{Introduction}

The aim of this paper is to obtain a strict form of propagation constant taking 
into account the noninertiality of the reference frame in which the value is determined. The rigidity of the form is determined by analytic approaches that do not exclude the required permissible approximations. The obtaining of a propagation constant for a non-inertial frame of reference in the region of a local source was described in [1] and [2]. This approach is inaccurate if we extrapolate the results to regions remote from the surface. Conversely, when approaching the source, the objective functions demonstrate catastrophic behavior (which is the standard behavior for singular equations). The analytic decomposition of the propagation constant into spatial harmonics (such as in [3] and [4]) does not allow one to interpret this value into the electrophysical target parameters, for setting up a computational experiment.

In fact, this work is a generalization of known facts in a complete methodology. The developed approach allowed eliminating the use of rest frame approximation in the laboratory. Nevertheless, in the boundaries of the work, some new data were obtained. Received new interpretation data that allow for high-precision modeling. This is of particular importance for the formulation of a computational experiment by the method of discrete elements in electrodynamics. Due to the mathematical features of the method, the accuracy of the calculation depends on the accuracy of the procedures for describing physical processes embedded in it. That is why obtaining a strict form is so important for this task. In this context, a special role is played by the problems of the propagation of electromagnetic waves in a plasma and modern methods for the solution ([5] and [6]).

A strict approach for obtaining a constant propagation in a translationally moving medium (inertial frame of reference) is described in [7]. The transition to a non-inertial frame of reference is analytically accompanied by great difficulties [8]. Therefore, for the first order of perturbations, rest frame theory is often used (such as in [3] and [4]). However, the perturbation approximation in order does not require the noninertiality to be neglected, if only because these effects also occur at nonrelativistic velocities (especially when performing sensitive measurements, which can be carried out now in the laboratory). Nevertheless, the analytical difficulties of taking into account the forces at each point of the space can increase the 
computational error in an unacceptable manner. The main problems were touched upon in the work [9]. To obtaining the expressions for propagation constant in moving media are also devoted the following works [10] and [11].

In this paper, we consider the following system: the monochromatic wave incidences on a rotating material medium represented in the form of a flat circular disk. The propagation of electromagnetic power occurs only in one plane corresponding to the plane of rotation. In this formulation, a two-dimensional electromagnetic propagation problem is solved in a non-inertial frame of reference. A rotating frame of reference is a good example of any noninertial frame of reference since it must take into account the action of inertial forces on any energy moving in it.

\section{Method}

The generalized geometry of the problem, applied to the method presented, is shown in fig. 1. Let us explain the notation introduced: $\Omega$ - angular velocity vector (direction of rotation counterclockwise), $R$ - radius of disk, $\alpha$ - angle between the incident ray and the perpendicular $\mathbf{n}$ to the point of incidence, $\xi_{\mathrm{cf}}$ and $\xi_{\mathrm{k}}-$ the angles of the ray deflection under the action of the centrifugal force and the Coriolis force, respectively. Other values will be explained below.

From the general provisions of the propagation of electromagnetic waves in noninertial reference frame follows that the inertial forces acting in the system (as well as the gravitational field in the inertial frame of reference) change the characteristics of space and spatial metric in a certain way [12]. A classical approach for describing the conditions for the propagation of electromagnetic waves in noninertial reference frames is the refined refractive index of the medium. For a vacuum located in an external gravitational field it is [13] and [14]:

$$
N_{U}^{g r}=1+\frac{2 U}{c^{2}},
$$

where $U=W_{p} / m$ - force potential with $W_{p}$ is a potential energy of a body with mass $m$ in a force field. Expression (1) is inaccurate since it does not take into account the change of the field in the space and time. Strictly speaking, formula (1) is universal 
and can be applied to any system with an external force field or equivalent system. In the Euclidean space, the deflection of the rays is described by the refraction refined above (this is the simplest approximation, although it does not correspond to physical processes).

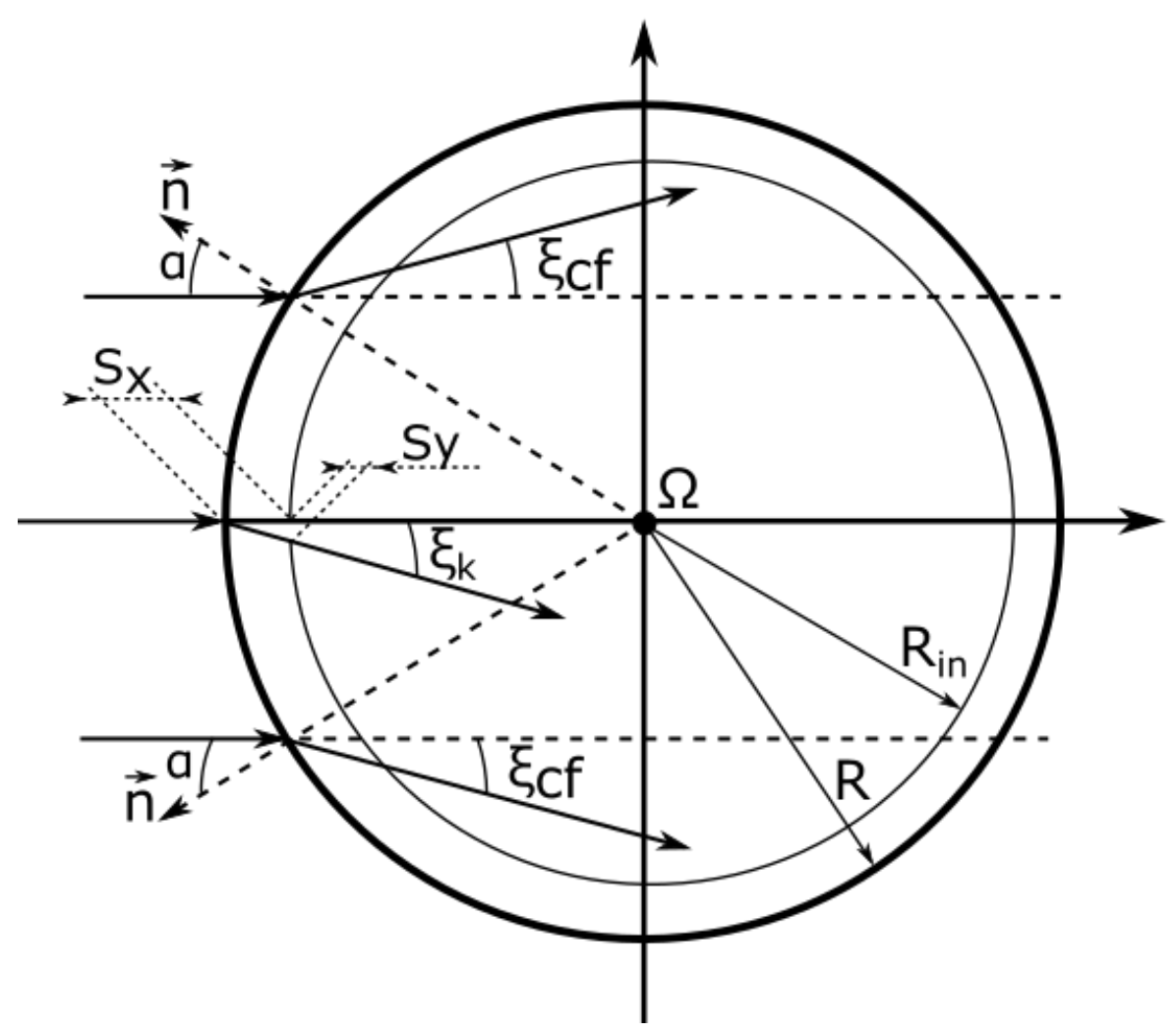

Fig.1. System construction geometry.

By the principle of equivalence, we can perform similar procedures for a rotating material system of points. A detailed comparison of reference frames in an external gravitational field and with constant acceleration is presented in [15]. We introduce a rotating frame of reference and a material body at rest relative to it. To convert (1) to rotating systems, it should be noted that the gravitational force is a conservative force and creates a potential field. Its action is directed toward the center. Centrifugal force is also conservative, however its direction is opposite - from the center, hence $\mathbf{F}_{\text {cf }}=$ $\mathbf{F}_{\mathrm{gr}}$. By definition, we have $F_{c f}=-\mathrm{d} W / \mathrm{d} R=m \Omega^{2} R \rightarrow \mathrm{Wp}=-0.5 m \Omega^{2} R^{2}$. Coriolis force is gyroscopic and cannot be described by a potential field, which is simply not created. Thereof, 


$$
N_{U}^{c f}=1-\frac{\Omega^{2} R^{2}}{c^{2}}=1-\beta^{2} .
$$

If we use the analogy with the lens, then the external gravitational field in vacuum - a converging lens with a real focus (the medium is more optically dense than just a vacuum), and rotating matter with vacuum parameters - a diverging lens with an imaginary focus (optically less dense than the medium, but at rest). The arising contradiction associated with the ratio of the phase velocities when the ray passes from an optically denser medium to an optically less dense medium is easily resolved by indicating that the rotational movement according to SRT can be implemented only to a material body with its own refractive index at rest: $N^{0} \geq 1+\beta^{2}$.

The resulting inequality is a physically reasonable limitation for the accuracy of determining the refractive index and was not met by the author earlier in open sources. An interesting fact is that a solid body that is at rest in any reference system cannot have a priori information about the angular velocity with which it will rotate in the future. Perhaps this observation is a confirmation of the Ehrenfest paradox, about the impossibility of giving a rotational motion to a solid body. On the other hand, this inequality is confirmed by the non-invariance of Maxwell's material equations with respect to the chosen reference system.

Now, we will obtain the value of the angle of deflection of the ray $\xi_{\mathrm{cf}}$, caused by the action of the centrifugal force. The medium is not spherically symmetric with respect to the coordinate dependence $N_{U}^{c f}(\rho)$ [16]. As a primary model, we may consider the equivaleposition of two centers of mass - they would be located symmetrically relative to the principal axis. In this case, we would consider two spherically symmetric system, so the ray going in the lower semicircle is a mirror image of the ray going in the upper semicircle and vice versa. However, such a system is not a good approximation because of the fundamental difference in the coordinate dependence of the index of refraction, so $N_{U}^{g r}(\rho) \propto \rho^{-1}$ and $N_{U}^{c f}(\rho) \propto \rho^{-2}$. It is not difficult to use the formulation of a generalized eikonal for a 
known dependence $N_{U}^{c f}(\rho)$, however, in the proposed method, this approach is redundant.

Just as in [14] refraction laws were obtained for a thin layer selected in a spherically symmetric medium, through the Snell's law, we isolated the inner layer the circle with the center at the origin and the radius $R_{\text {in }}$ and given that $R_{\text {in }} \rightarrow R$. At the radius of the layer $R$, the maximum inertia force acts on the energy, but at the radius of the layer, $R_{\text {in }}$ the inertial forces are zero. Thereby,

$$
\xi_{c f}=\arcsin \left(\frac{\sin \alpha}{N_{U}^{c f}}\right)-\alpha .
$$

Next, find the angle $\xi_{\mathrm{k}}$. The deflection of the ray from a rectilinear trajectory in dynamics is related only to the action of the Coriolis force. Therefore, we can indicate $\tan \left(\xi_{\mathrm{k}}\right)=s_{y} / s_{x}$, where $s$ - moving the ray along the corresponding axes in time $\Delta t$. For the $s_{y}$, we have: $s_{y}=\left(a_{k} \Delta t^{2}\right) / 2$ and $s_{y}=\Omega s_{x} \Delta t$, where $a_{k}$-acceleration due to Coriolis force. Equate these two expressions, we also consider that $a_{k}=F_{k} / m$, where $\mathbf{F}_{\mathbf{k}}=2 m\left[\mathbf{\Omega} \times \mathbf{V}_{\mathbf{p}}\right]$ and $\mathbf{V}_{\mathbf{p}}-$ phase velocity in a medium. As a result, we obtain $s_{x}=V_{p} \Delta t \sin (\Psi)$, where $\Psi-$ angle between the vectors of angular velocity and phase velocity. The target value becomes $\tan \left(\xi_{\mathrm{k}}\right)=\left(\Omega s_{x}\right) /\left(V_{p} \sin (\Psi)\right)$. In order for the resulting expression to become applicable for the calculation, it is necessary to solve three problems. First - unknown value of $s_{x}$, second - the value of the phase velocity of the wave in the medium and the last one - unknown angle $\Psi$. In order to solve these problems, it is necessary to introduce a number of approximations and conventions.

It was obtained that the linear measurement of this sector should be equal to $s_{x}=R \beta$. The justification of this identity is given in [1]. We also assume that all inertial forces act on electromagnetic energy only on this part of the path. The phase velocity will be sought through the Fresnel coefficient of $\operatorname{drag} F_{p}$, if one needs to be taken into account [17]. We will assume that the propagation of an electromagnetic wave is always in the plane of rotation, and hence $\Psi=\pi / 2$. As a result, we have 


$$
\xi_{k}=\arctan \left(\frac{v \beta}{V_{p}^{0}+v F_{p}}\right)
$$

where $v$ - linear velocity of the reference frame, $V_{p}^{0}-$ phase velocity of a wave in a medium without rotation. Expressions for phase velocities in a moving medium were obtained in the papers [10] and [11], which can also be used here.

Let us find the full path $S$ of the ray under the action of inertia forces. From the above assumptions it follows that $S=\sqrt{s_{x}^{2}+s_{y}^{2}}$. Substituting the values of the quantities and taking into account that $\Delta t=s_{x} / V_{p}$, we have $S=R \beta \sqrt{1+\left(\frac{v \beta}{V_{p}^{0}+v F_{p}}\right)^{2}}$. This value does not depend on one coordinate in the system, and therefore remains constant for any ray coming from any direction. Therefore in the system, the internal circle, as the horizon of action of forces of inertia is formed. The radius of this circle is $R_{\text {in }}=R-S$, or

$$
R_{i n}=R\left(1-\beta \sqrt{1+\left(\frac{v \beta}{V_{p}^{0}+v F_{p}}\right)^{2}}\right)
$$

The expression (5) is also not previously encountered in open sources. The simplicity of the conclusion does not diminish the importance of using the action horizon of inertia forces, as a special layer in the process of stratification, in which the description of the electro-physical parameters of the medium should be implemented with increased accuracy.

When calculating the wavenumber in the rotating system, it is necessary to take into account the frequency shift caused by noninitiality. Variation of the frequency occurs when energy is transferred from a region with a large force potential to an area with a smaller force potential, and the appearance of this effect is associated only with the centrifugal force. The total energy of the quantum changes and this is equivalent to a change in its frequency [14]. We have $\Delta f=\Delta U / h$, where $h-$ Planck's constant. We also assume that the ray propagates to a region with zero potential, 
hence $\Delta U=0.5 m \Omega^{2} R^{2}$. We will not express the mass of electromagnetic energy through its frequency since it can be unknowable if the material medium is connected with the reference frame. We use the concept of volume energy density contained in a given space at a given time $\omega_{\mathrm{emf}}=\omega_{\mathrm{e}}+\omega_{\mathrm{m}}$, where $\omega_{\mathrm{e}}$ and $\omega_{\mathrm{m}}-$ volume energy density of electric and magnetic fields. By definition, the energy of the electromagnetic field is $\mathrm{W}=\omega_{\mathrm{emf}} \Delta \mathrm{V}$, where $\Delta \mathrm{V}-$ volume unit. From the provisions of electro and magnetostatics can specify $\omega_{\mathrm{emf}}=\varepsilon \varepsilon_{0} E^{2}$, where $E$ - electric field strength. As the result:

$$
m=\frac{\varepsilon \varepsilon_{0} E^{2} \Delta V}{c^{2}}
$$

The final formula for the frequency shift:

$$
\Delta f=\frac{\varepsilon \varepsilon_{0} E^{2} \Delta V \beta}{2 h}
$$

Explanations and alternative approaches can be obtained in work [18].

Now we get the full angle of refraction of electromagnetic waves in a rotating material medium $\Xi$. Let us turn again to the fig. 1. A ray incident on the interface between two media is naturally refracted relative to the perpendicular vector $\mathbf{n}$. Denote the angle of natural refraction, as $\theta$. Assuming that the incident wave propagates in a vacuum, from Snell's law we have: $\theta=\arcsin \left(\sin \alpha / N^{0}\right)$. Consequently, in the first quadrant: $\Xi=\theta+\xi_{\mathrm{cf}}-\xi_{\mathrm{k}}$, in the second quadrant $\Xi=\theta+\xi_{\mathrm{cf}}$ $+\xi_{k}$. Summarizing:

$$
\Xi=\arcsin \left(\frac{\sin \alpha}{N^{0}}\right)+\xi_{c f} \pm \xi_{k}
$$

The paper [1] shows the decomposition of the constant propagation along the coordinates of the system. In this context, we are interested in a strict record. The solution of the wave equation is the combination $\gamma=\operatorname{Re}\left(\gamma_{+}\right)+\operatorname{jIm}\left(\gamma_{-}\right)$, where 


$$
\begin{aligned}
& \gamma_{ \pm}=\frac{-C(\rho) \pm \sqrt{C(\rho)^{2}+4\left(k^{0}\right)^{2}}}{2}, \\
& C(\rho)=\Omega \rho \sin \Xi\left(2 \omega^{1} M+j \mu \sigma\right), \\
& M=\frac{\left(N^{0}\right)^{2}-1}{c^{2}}, \\
& k^{0}=\sqrt{\frac{\left(\omega^{0}\right)^{2} \varepsilon_{r}}{c^{2}}+j \omega^{0} \mu \sigma,} \\
& \omega^{0}=2 \pi f \rightarrow \omega^{1}=2 \pi(f+\Delta f) .
\end{aligned}
$$

We consciously avoid the decomposition of the propagation constant into spatial harmonics, because in this case there is no way to obtain a strict distribution of the value inside the frame of reference. Allowance for the angle of total refraction $\Xi$ in the expression for the coordinate function $C(\rho)$ extends the description of the system beyond the Rest frame theory, which is actively used in semi-analytical considerations [3], [4] and [8].

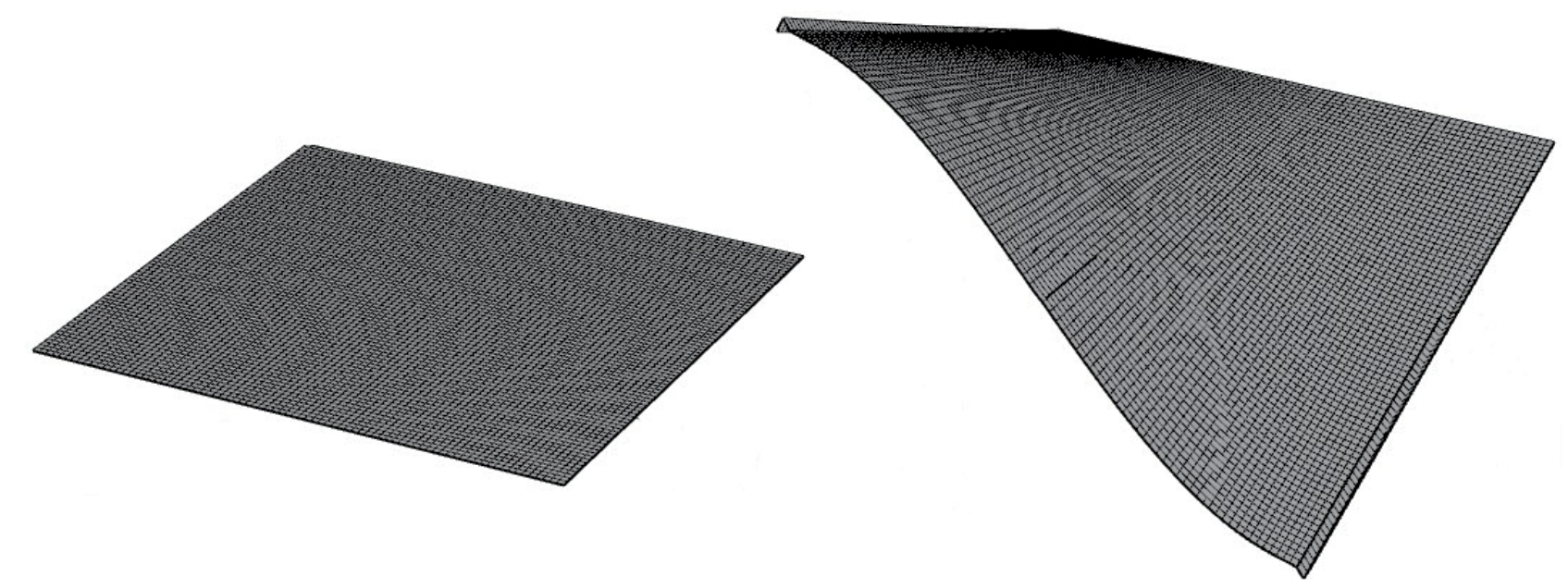

Fig.2. A curvature of the wave vector space caused by rotation of the reference frame.

As an example, fig. 2 shows the curvature of the spatial distribution of the propagation constant in the rotating frame of reference. On the left is shown the three dimensional distribution of $\gamma$ in one semicircle of the system without rotation, on the right the plane with the nonzero angular velocity. A key feature in the space of the wave vector associated with the non-inertiality of the system is the appearance of a 
local extremum in the distribution of the value. In fig. 2, these are features for which the smoothness of the surface is lost.

\section{Results}

To obtain and verify the results, we use the following input parameters for calculations: $f=200 \mathrm{MHz}, a=0.955 \mathrm{~m}, \mu_{\mathrm{r}}=1-0 \mathrm{j}, \varepsilon_{\mathrm{r}}=4-0.2 \mathrm{j}(\sigma=0.00223 \mathrm{~S} / \mathrm{m})$ and $\Omega=9.3 \times 10^{6} \mathrm{rad} / \mathrm{s}$. For ease of analysis, these data completely correspond to the parameters of the analytical calculation in [4].

First of all, as the grossest approximation, we considered a variant in which the wave vector in the medium and the vector of the linear velocity of the medium itself do not form an angle. This situation was the result of an analysis of the translational motion of the medium considered in the paper [7]. It should also be pointed out that in the absence of inertia forces, there should be no (or compensated) natural refraction at the interface between the two media. In this case, we obtain the distribution of the propagation constant, refined only and exclusively by the drag coefficient $F_{p}$. In this case, we are only interested in a confirmation of the correctness of the proposed formulations. The distribution of the real and imaginary parts of $\gamma$ is shown on the fig. 3. In this case, it is obvious that the propagation constant depends only on the radius, and linearly. The magnitude of the magnitude varies uniformly from the center to the surface of the rotating body. The general picture of the space of the wave vector is shown in fig. 4. This distribution was obtained directly by calculating the function (9).

The contours of the distribution of the $\operatorname{Re}(\gamma)$ at fig. 4 are approximated by a second-order basis function. However, as the study showed, the approximation by a first-order basis function is also acceptable, especially at low angular velocities, since the contours of the distributions are close to linear. The simulation of the system was carried out by the method of moments. To the cylindrical geometry, periodical boundary conditions were applied along the $\mathrm{z}$ axis, which coincides with the analytical representation of an infinite cylinder. The decomposed system is shown in fig. 5 . 


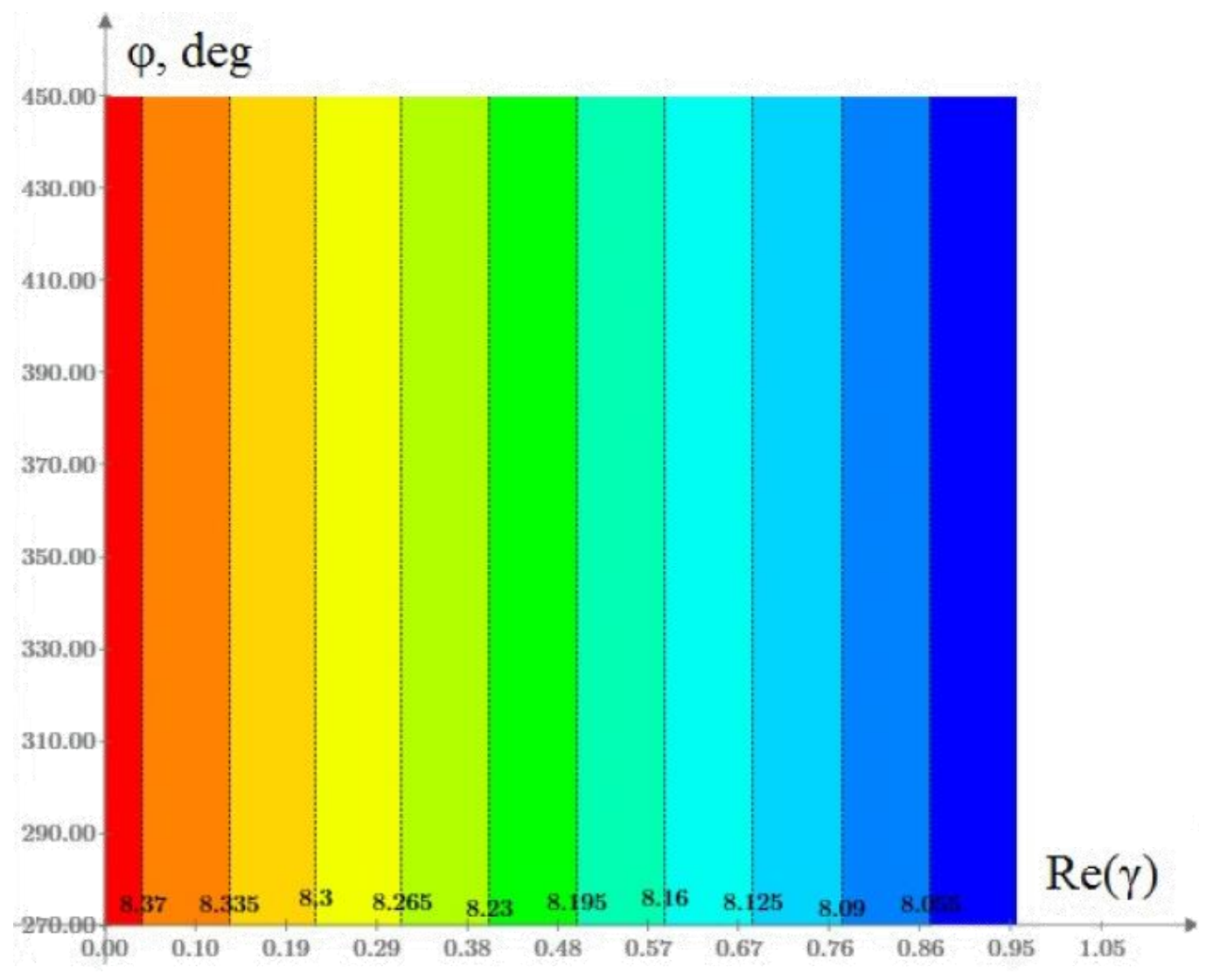

Fig.3. The contours of the distribution of the propagation constant with $\phi=0$.

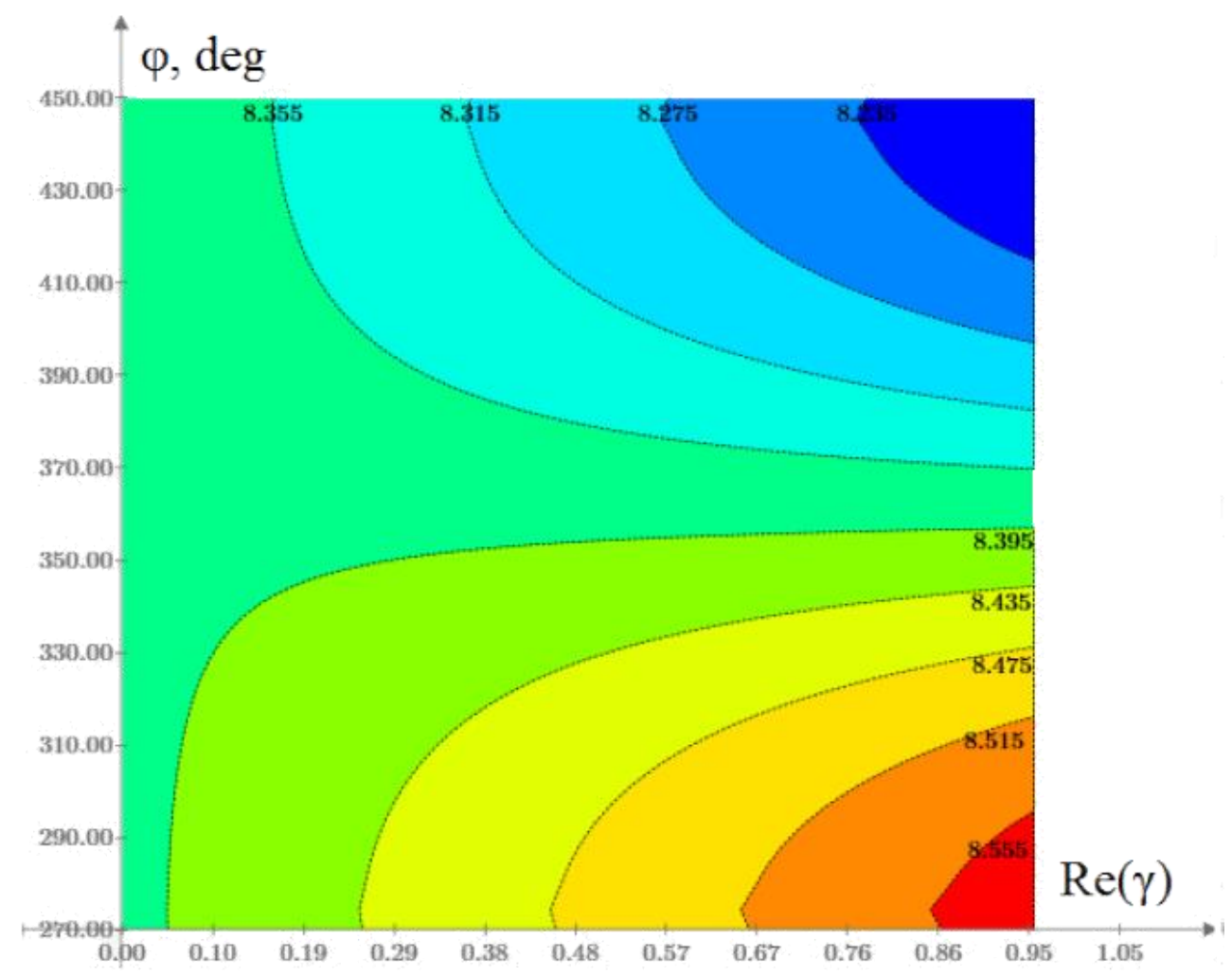

Fig.4. The contours of the distribution of the strict form of the propagation constant. 


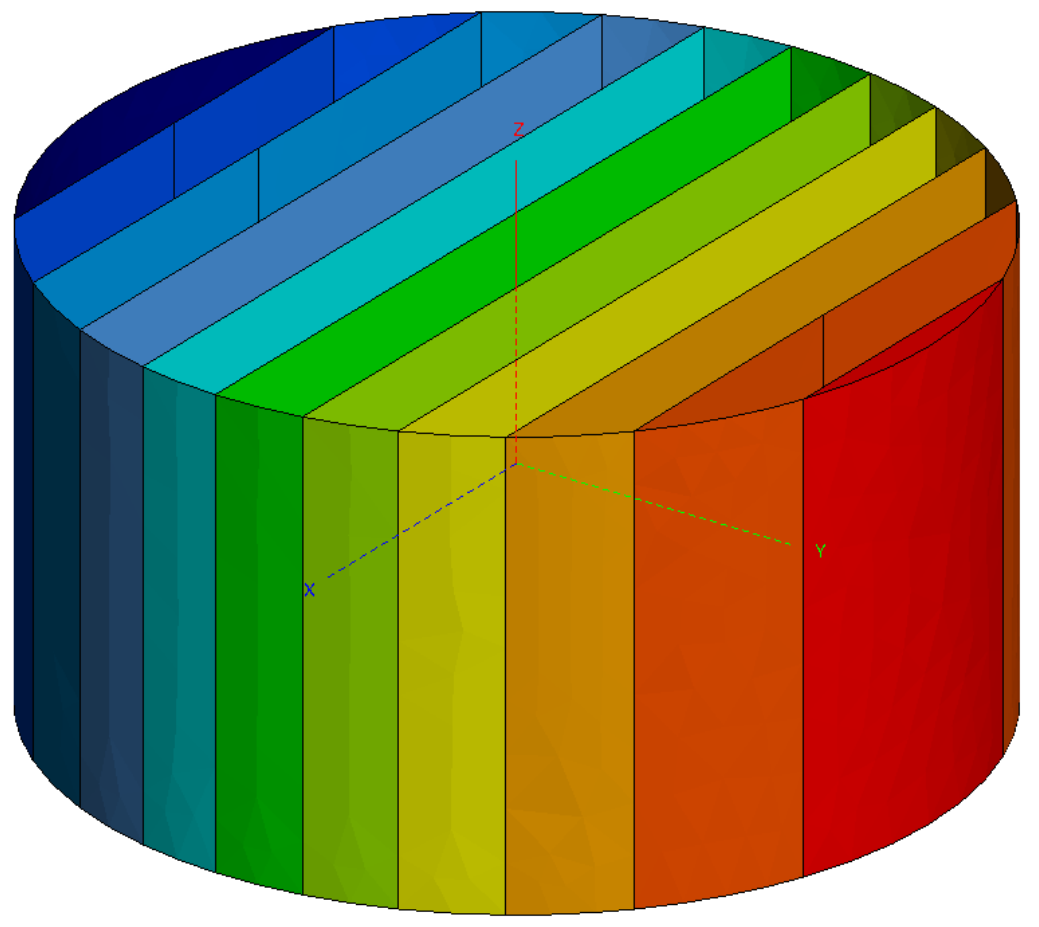

Fig.5. The simulated system decomposition.

Verification is carried out by reconciliation with the analytical data presented in [4], however, it should be emphasized that they are also theoretical. In the process of validation, a number of discrepancies were found, which, however, are also present in the method described in [1]. Dividing the resulting scattering pattern by the radiation into infinity region and backscattering, we obtained that, in order to correspond to the analytical data, the direction of rotation should be reversed. The region of the inverse radiation does not correspond to analytic expressions because the direction of propagation of the wave must be reversed. It is impossible to take this situation into account in the framework of one model. To obtain the scattering pattern over the entire horizontal plane, we can use the results-matching algorithm presented in [2]. To justify this algorithm, it is sufficient for us to perform a reconciliation in the region of radiation to infinity.

The resulting scattering patterns are shown in fig. 6. We give a complete scattering diagram, but verify the results only over the radiation to infinity region. It should also be noted that the analytical solutions do not take into account the actions 
of inertia forces, so there is no precise data for verifying the results. Nevertheless, for $\beta \rightarrow 0$, the results are reduced to complete uniformity, which allows us to make a conclusion about correctness.

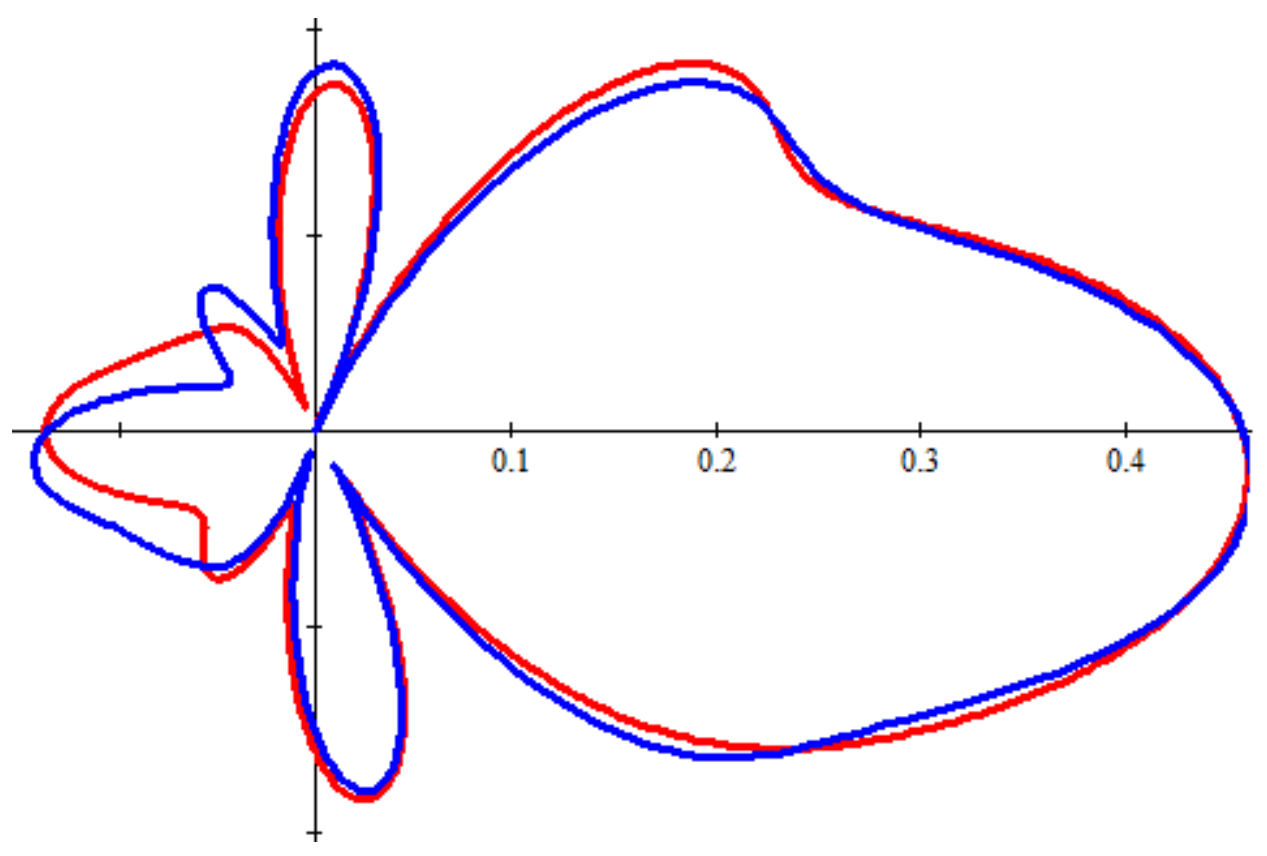

Fig.6. Comparison of numerical and analytical results (blue line - analytics and red line - modeling).

\section{Conclusion}

In conclusion, it should be pointed out that the proposed algorithm in the computation experiment is complete only when accounting for a computational error, which should be minimized by the mesh optimization. This study has already been carried out and described in work [20].

We can also point out that the use of a functional approach [21] in the description of the material media of noninertial frames of reference seems promising, which fits well into the context of decomposition techniques for numerical simulation (as in fig. 5).

In general, we can conclude that the synthesis of the propagation constant can be considered complete and rigorous in the framework of the proposed approach, but the lack of information for verification of results leaves a number of questions open. 


\section{References}

1. Zeyde K.M. The coordinate expression of the propagation constant for a moving dielectric medium. 2018 USBEREIT Conference Proceedings. Ekaterinburg, 2018, pp. 295-298 (In Russian).

2. Zeyde K.M. Fast segmentation of a rotating axisymmetric scatterer medium of an arbitrary form for the first order fields numerical analysis. Ural Radio Engineering Journal, 2018, Vol. 2, No. 2, pp. 26-39 (In Russian).

3. Van Bladel J. Electromagnetic fields in the presence of rotating bodies. Proceedings of the IEEE, 1976, Vol. 64, No. 3, pp. 301-318.

4. De Zutter D. Scattering by a rotating circular cylinder with finite conductivity. IEEE Trans. on antennas and propagate, 1983, Vol. AP-31, No. 1, pp. 166-169.

5. Cary J.R. Particle-in-cell methods for modeling electromagnetic propagation in plasmas. ACES Conference Proceedings. Denver, USA, 2018.

6. Li X., Li D., Yue H., Zhang Z., Liu K., Fan M., Chen D. A high precision particlemoving algorithm for particle-in-cell simulation of plasma. IEEE Trans. On magnetics, 2015, Vol. 51, No. 3.

7. Collier J.R., Tai C.T. Propagation of plane waves in lossy moving media. IEEE Trans. on antennas and propagation, 1964, Vol. 12, No. 3, pp. 375-376.

8. Irvine W.M. Electrodynamics in a rotating system of reference. Physica. 11964, Vol. 30, pp. 1160-1170.

9. Stephenson L.M. Relativistic electromagnetic solutions in rotating systems. Proceedings of the IEEE ,1966, Vol. 54, No. 6, pp. 885-886.

10. Nicolis J.S., Lin C.C. Refractive index and group velocity in moving dispersive media. Radio Science, 1970, Vol. 5, No. 3, pp. 625-634.

11. Shukla P.K., Singh R.P., Singh R.N. Refractive index of drifting plasma. IEEE Trans. on antennas and propagation, 1971, Vol. 19, No. 2, pp. 295-296.

12. Volkov A.M., Izmest'ev A.A., Skrotskii G.V. The propagation of electromagnetic waves in a Riemannian space. Journal of Experimental and Theoretical Physics, 1971, Vol. 32, No. 4, pp. 686-689. 
13. Plebanski J. Electromagnetic waves in gravitational fields. Physical Revier, 1960, Vol. 118, No. 5, pp. 1396- 1408.

14. Yakovlev O.I. Rasprostranenie radiovoln v kosmose [Propagation of radiowaves in cosmos]. Moscow, Nauka Publ., 1980 (In Russian).

15. Acedo L., Tung M.M. Electromagnetic fields in a uniform gravitational field and Plancks postulate. European Journal of Physics, 2012, Vol. 33, No. 5.

16. Solimeno S., Crosignani B., DiPorto P. Guiding, diffraction and confinement of optical radiation. Academic Press, Inc., 1986.

17. Berger H., Griemsmann J.W.E. Moving media without electromagnetic drag. IEEE Trans. on antennas and propagation, 1967, Vol. 15, No. 4, p. 585.

18. Liang-Cheng Tu, Jun Luo, Gillies G.T. The mass of the photon. Reports on Progress in Physics, 2005, No. 68, pp. 77-130.

19. Shiozawa T. Phenomenological and electron-theoretical study of the electrodynamics of rotating systems. Proceedings of the IEEE, 1973, Vol. 61, No. 12, pp. 1694-1702.

20. Zeyde K.M. Setting and physical rationale of heterogeneous turning scatterer system mesh optimization. ICECom Conference Proceedings. Dubrovnik, Croatia, 2016.

21. Starke R., Schober G.A.H. Microscopic theory of refractive index. International Journal for Light and Electron Optics, 2017, Vol. 140, pp. 62-85.

\section{For citation:}

K. M. Zeyde. The complete form of the propagation constant in a noninertial reference frame for numerical analysis. Zhurnal Radioelektroniki - Journal of Radio Electronics. 2019. No.4. Available at http://jre.cplire.ru/jre/apr19/3/text.pdf

DOI $10.30898 / 1684-1719.2019 .4 .3$ 